\title{
DETERMINING THE GEOMETRICAL ELEMENTS IN THE PHOTOGRAMMETRIC METHOD FOR MEASURING LINEAR DIMENSIONS
}

\author{
Branko Sotirov, Danko Tonev, \\ Svetlin Parvanov \& Miroslav Kokalarov
}
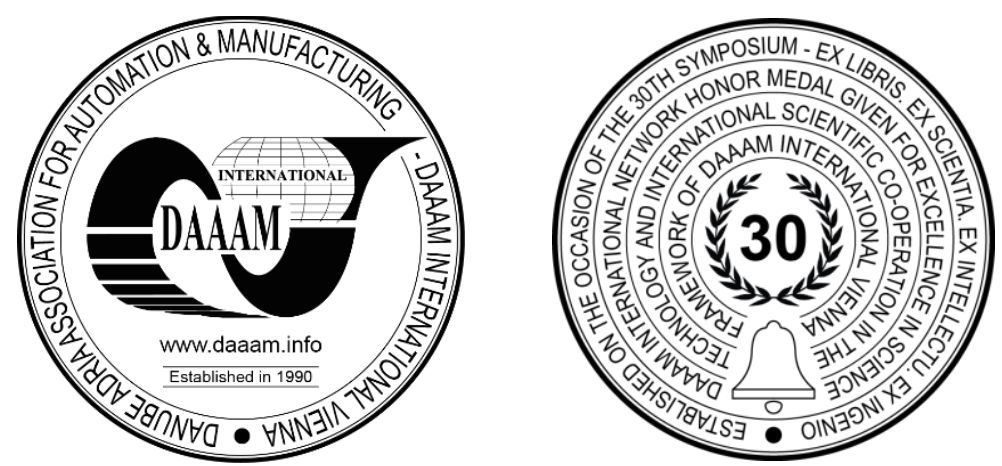

This Publication has to be referred as: Sotirov, B[ranko]; Tonev, D[anko]; Parvanov, S[vetlin] \& Kokalarov, M[iroslav] (2021). Determining the Geometrical Elements in the Photogrammetric Method for Measuring Linear Dimensions, Proceedings of the 32nd DAAAM International Symposium, pp.0275-0280, B. Katalinic (Ed.), Published by DAAAM International, ISBN 978-3-902734-33-4, ISSN 1726-9679, Vienna, Austria

DOI: $10.2507 / 32$ nd.daaam.proceedings.039

\begin{abstract}
This paper presents the specific characteristics of the photogrammetric measurement method applied in industry. The focus of the study are linear and angular dimensions of mechanical parts from heavy industry. The definitions of sizes to be determined using the photogrammetric measurement method are based on the international standards ISO 144051:2016 and ISO 286-1:2010 dealing with geometrical product specifications. The main objective is to define the algorithms for processing the results obtained by the photogrammetric method, and to use the method without the need of a specialized software which is costly and limits the applicability of the method in small and medium-sized companies.
\end{abstract}

Keywords: photogrammetry; photogrammetric measurement method; the direct global associated size; direct global size.

\section{Introduction}

Photogrammetry is an applied science used widely in various areas of metrological practice - construction, architecture, geology, agriculture and forestry, mapping, mechanical engineering, historic research, documentaries, etc. It is known that some of the most critical and frequently controlled parameters in heavy manufacturing are the deviations of linear and angular features of size (dimensions), form, location and orientation of surfaces and axes.

Even though the photogrammetric measurement method (PMM) is relatively new in respect to its application in mechanical engineering, it widens its application in manufacturing technologies and reengineering, where it is used for measuring small and very small, as well as large (more than $500 \mathrm{~mm}$ ) measured objects. It is known that there exist specialized methods, equipment and software for such measurements. They develop continuously but currently their high prices present a serious obstacle to their distribution in Bulgaria [5], [6]. It is established that when controlling small dimensions of large structures, the total measurement uncertainty shall not exceed $10 \div 20 \mu \mathrm{m}$, whereas for large dimensions it may be several times greater. At the same time, there is no sufficient information about the methodology for processing measurement information related to determining the necessary geometrical elements and parameters of the measured objects. 
This methodology is directly related to the accuracy and reliability of the results obtained and immediately affects the measurement quality of the photogrammetric method, therefore, affects the quality of the assessed products.

\section{Linear distance in the photogrammetric measurement methods}

The photogrammetric measurement method (PMM) is classifies within the group of contactless methods that best illustrate (visually) terms such as 'integral feature', 'real feature', 'extracted integral feature', 'associated integral feature' [4]. In the case of PMM, these terms characterize the existence and the type of a specific repeating fragment in the image. The subjective factor plays a significant role in the interpretation of the limits/contours of measured objects made of different materials (metallic and non-metallic).

The presence of a texture that characterizes the existence and the type of a specific repeating fragment in the image depends on the sizes of identical objects that produce it and on the scale of the image, respectively. This allows to differentiate objects that cannot be defined otherwise, because practically they blend into the background. Fig. 1a presents a fragment of the contour of a metallic object, shot a close range, and Fig. 1b - presents a fragment of the contour of a non-metallic object, both images magnified 2400 times. The figure clearly demonstrates that even though under normal magnification a line theoretically is described, in the worst case, by 3 px, in reality, especially for non-metallic materials, this becomes more complicated. When the photographic image is greatly enlarged, the constituent pixels can be clearly seen.

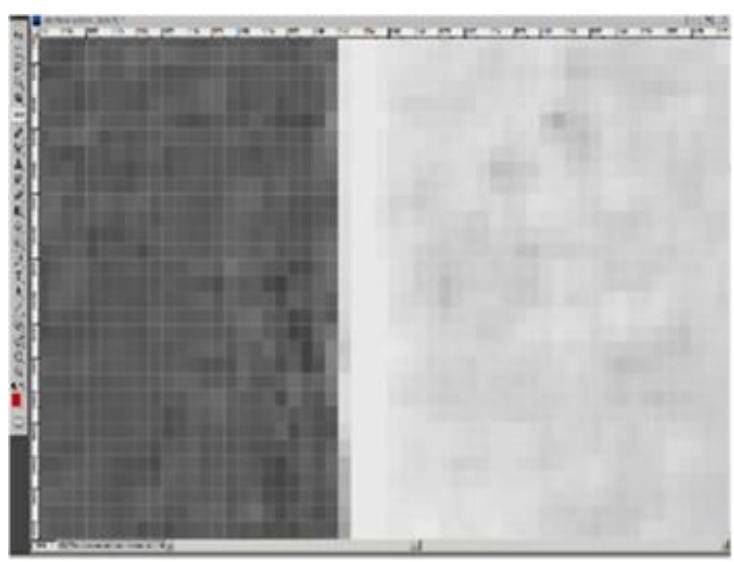

a)

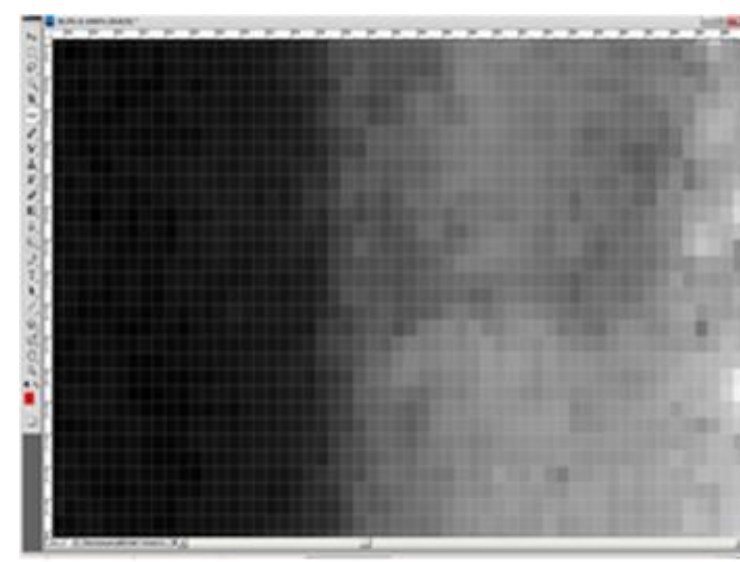

b)

Fig. 1. A fragment of the contour of: a) a metallic object, and b) a non-metallic object

The main reason for this fact is the circumstance that the dimension, determined using PMM, and respectively 'industrial photogrammetry', is the result of a quantitative piece of information, obtained from a multitude of pixels of a photographic image of the measured object. This finite number of pixels determines the extracted integral feature, obtained by taking a photo. It is known that the photogrammetric determining of geometrical dimensions is a combination of two activities - taking a photo and measuring. In this sense, the dimension determined using the PMM has to be defined as a direct global size, obtained as associated in a direct measurement method [1], [3], [5] (Fig. 2).

In certain isolated cases, the discussed dimension conditionally could also be defined as indirect global size, calculated from a homogeneous group of local dimensions. The main problem in this case is to justify the definition of local dimensions, obtained by using PMM. The direct global associated size (DGAS) is a geometrical feature of a nominal surface approximating (associated with) a real surface. Therefore, DGAS can be placed within the material of the measured object or outside of it, whereby the nominal surface is enveloping the real profile. It is a unique measurement and assessment result that eliminates the influences of the form, location and orientation of the object [2].

In the first case, the DGAS is a mean feature obtained from the extracted integral feature (profile) using the leastsquares association criteria. It is known that this method provides stable and easily reproducible results [2]. In the second case, the DGAS can be obtained as the maximum inscribed size, the minimum circumscribed size or as a minimax size of the extracted integral feature (Fig. 2). It is known that the envelope requirement is the ideal form for maximum material sizes. The choice between the two approaches is determined mainly by the exploitation purpose of the measured surface, the requirements and/or the possibility for assembly, etc.

\section{Specifics of determining sizes in the photogrammetric measurement method}

The specifics of determining sizes are defined by the geometrical form of the measured object. In mechanical engineering, the generalized form of object in respect to their measurement can be cylindrical, cuboid or, relatively less frequently - spherical form, regardless of their size- small, average or large. Any of the generalized cases mentioned above has features that are strictly characteristic of it. 


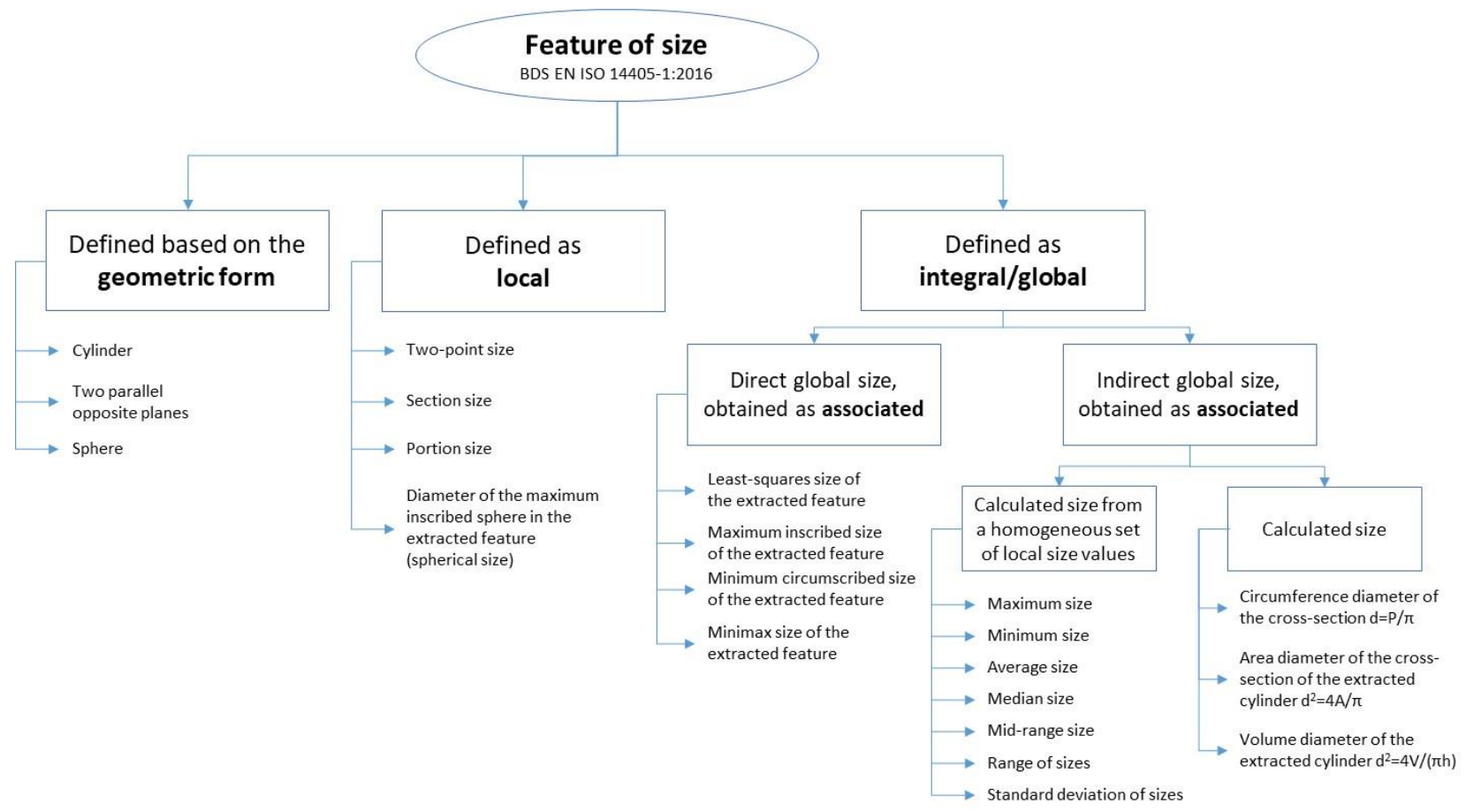

Fig. 2. Defining of features of size

\subsection{Specifics of developing the contour description of the image in the photogrammetric measurement method}

As mentioned in item 2, PMM is a contactless method that is applied on a photograph (image) of the object/part having a reading resolution of 1 px by using software. The developing of a contour description of the image can be obtained by segmentation of the image area into areas and by determining the contours in the image. It is based on the assumption that the individual areas are separated by a more or less pronounced boundary. On the two sides of this boundary can be seen a sharp change in the parameters of the image (for example, dark-light). This change may also manifest itself by a change in the average grey level or of the statistical parameters that describe in colour the texture areas. The contour is the line where the change in the parameters occurs. Depending on the character of this change one can see edges or a zone which contains the edge. The edge is a boundary which separates areas with different properties [8].

This change in the characteristics, observed in the normal direction to the boundary line, is approximated with a sharp transition - a step (Fig. 3a), ideally two adjacent pixels) or a smooth transition (Fig. 3b), in reality, a minimum of 3 pixels). The situation is even more complex because of the lack of sharp edges and the presence of relatively large rounding radii at the ends of the measured surface (Fig. 3). This change in the characteristics, observed in the normal direction to the boundary line, is approximated with a sharp transition - a step (Fig. 3a), ideally two adjacent pixels) or a smooth transition (Fig. 3b), in reality, a minimum of 3 pixels). The situation is even more complex because of the lack of sharp edges and the presence of relatively large rounding radii at the ends of the measured surface (Fig. 3).

Based on the experience gained to date it is known that the measurement uncertainty is significantly influenced by the parameters of the photographed object, the parameters of the matrix and the parameters of the optical system of the camera used. Under a certain unfavourable circumstances, their influence may be decisive for the application of PMM. The results of experimental studies demonstrate that the measurement uncertainty increases significantly in case of poor focusing combined with shading and large rounding radii, regardless of image processing and the software used [6], [8]. The main reason for this is the subjective interpretation / assessment of the boundary of the measured object. This boundary is the visualized (on the computer display) thickness of the marker line of the software used. In this context, it is influenced by the positioning (aiming) error of the start marker or the end marker in the process of defining the measurement distance between the pixels of the display [9]. This significant circumstance requires the development of a justified approach for determining the specific feature of size, and then its value from the image in PMM.

\subsection{Determining linear features of size of cylindrical parts}

In this case, the part is defined by two basic dimensions - the diameter of the cross-section, and the length of the generatrix of the cylinder. The diameter of the cross-section in the case of a $2 \mathrm{D}$ photogrammetric measurement can be determined only in the two end sections, and in the case of a 3D measurement - in a random number of cross-sections. Finally, the required diameter can be calculated as the average value of the measured values. 


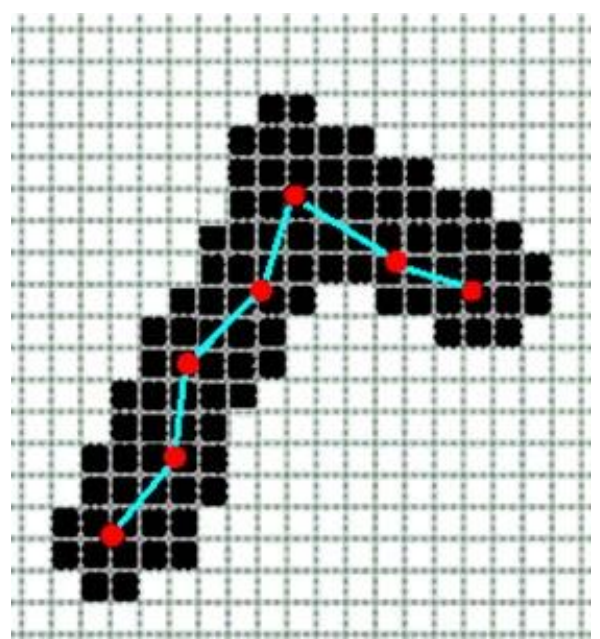

a)

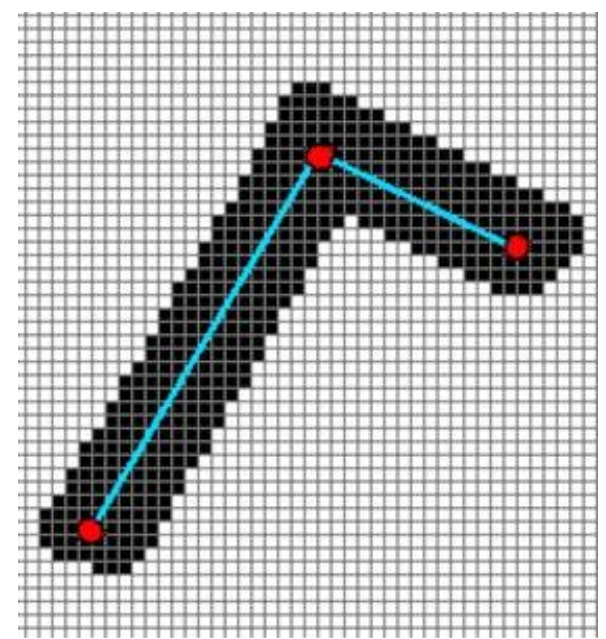

b)

Fig. 3. Forming a contour image: a) real, b) ideal

The determining of the diameter of a cylindrical part in an end section as a DGAS is performed in the following sequence: a) determining the pixels having the smallest and the largest radius (the outermost and the innermost point of the extracted feature) (Fig. 4). These pixels have the greatest potential for outliers (gross errors), thus they are excluded from the calculations. Naturally, in this case one can use a standard criterion for discovering outliers, which is applied to pixels located about the average of value of the coordinated of the obtained $\mathrm{N}$ pixels;

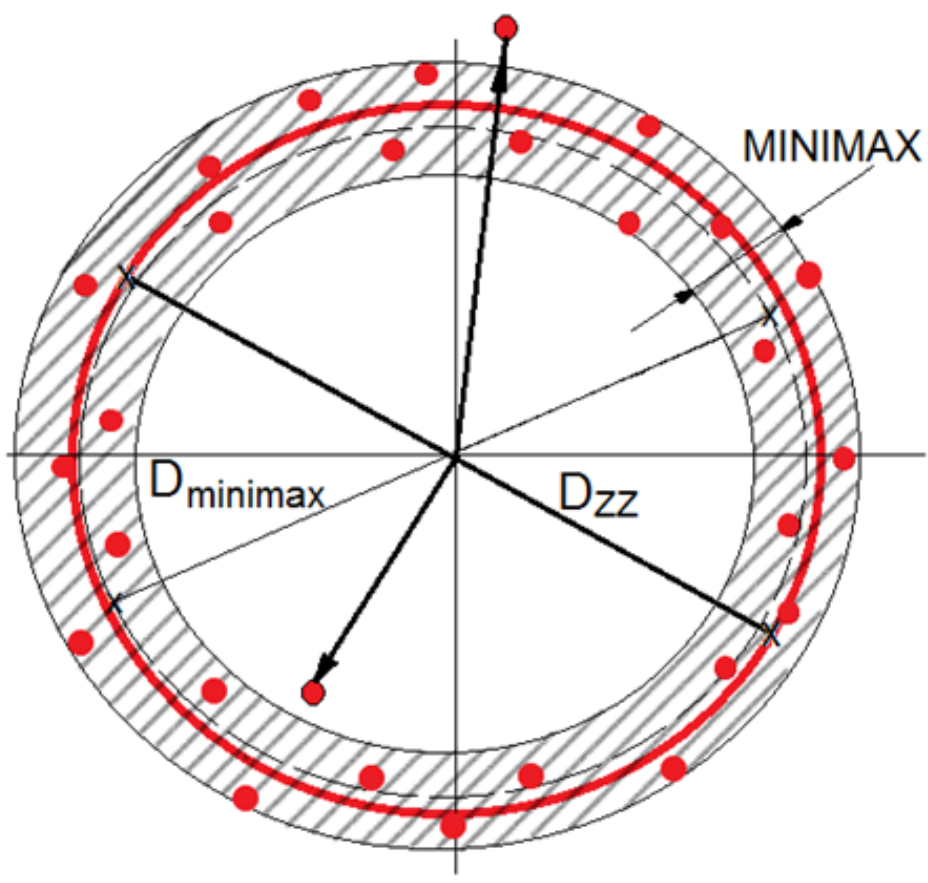

Fig. 4. Diameter of a cylindrical part

b) building a mean circle for the remaining N-2 pixels by applying the least-squares association criteria. The diameter of the circle $\mathrm{D}_{\mathrm{zZ}}$ is the required direct global associated size of the diameter of the measured cylindrical object;

c) if the dimension is determined graphically, the mean circle can be built based on the middle of the dimension of the minimal zone MINIMAX of the extracted integral feature, and the required diameter Dminimax is the distance between two opposing pixels, obtained by the intersection between the mean circle and a line passing through the centre of the extracted feature (Fig. 4). The application of this approach does not require neither the use of specialized photographic equipment, nor the use of specialized software.

d) if the dimension being determined is part of a fit, the required diameter is determined as the diameter of the largest inscribed circle $\mathrm{D}_{\mathrm{GX}}$ of the extracted integral feature for holes (internal features of size) or the diameter of the smallest circumscribed circle $\mathrm{D}_{\mathrm{GN}}$ of the extracted integral feature for shafts (external features of size).

The length of the generatrix of the cylindrical part can be determined by following the methodology, described in item 3.3 below. 


\subsection{Determining linear features of size of cuboid parts}

The discussed measurement object most often are parts having the form of a rectangular parallelepiped (cuboid) and dimensions up to 500 (3150) $\mathrm{mm}$ or prismatic-housing parts with average or large dimensions (up to and more than 10000 $\mathrm{mm}$ ), used in heavy machinery. In this case, the most frequently measured dimensions are linear distances between surfaces, which nominally are parallel to each other, and often are nominally parallel and/or perpendicular to other datum surfaces. The linear distance between the associated features of two nominally parallel surfaces is the DGAS. It is determined in the following sequence:

a) Build a mean straight line for the photographed (visualized) pixels of the extracted integral feature (surface) by applying the least-squares association criteria. Identify the two extreme pixels and exclude them from further calculations. Here, similar to item 3.2 a), one could also apply a standard criterion for discovering outliers. After eliminating the outliers, by applying the least-squares association criteria once again determine the final mean straight line of the extracted integral feature (Fig. 5). Objectively considered, the resulting associated straight line may not fulfil the requirements for deviation from form and/or location in relation to the datum surfaces.

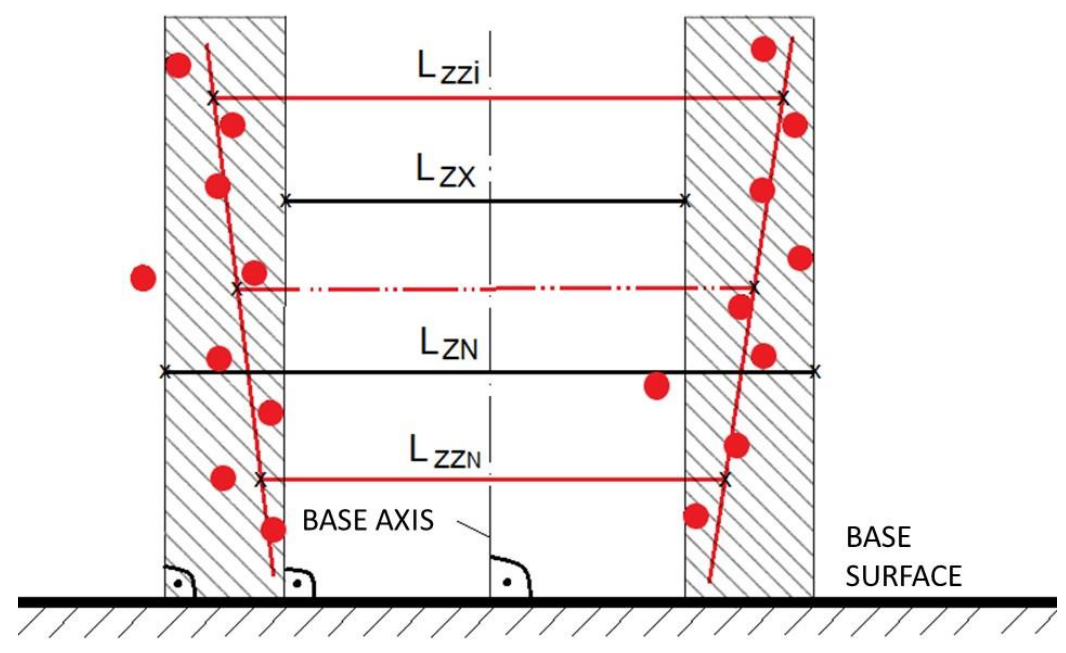

Fig. 5. Direct global size of a cuboid part

The latter are used for positioning the axis of symmetry (or another datum axis) between the parallel surfaces of the nominal feature;

b) The procedure for determining the mean straight line is repeated for the pixels of the extracted integral feature that describe the opposite, nominally parallel surface. The obtained second straight line probably will not be parallel to the first associated mean straight line, determining the first pixel of the required dimension. Consequently, it also may not fulfil the requirements for deviation from form and/or location in relation to the datum surfaces, indicated on the nominal features of size - the part drawing.

This circumstance allows to determine some deviations from form and/or location in relation to the datum surfaces.

c) Build a datum axis that meets the requirements for parallelism or perpendicularity of a specified nominal datum surface. It is appropriate that the datum surface is built as an enveloping surface of the extracted integral datum feature. If such a datum is not specified, then for the purposes of measurement it is necessary to establish a simulated datum feature [4]. A possible solution when measuring cuboid parts is to use a real, precise enough surface, that is perpendicular or parallel to the measured ones, and is associated to a straight line built according to the least-squares association criteria or as an enveloping surface;

d) If the measured dimensions belong to surfaces that are part of functional fits, instead of mean straight lines one has to use the maximum inscribed size $\mathrm{L}_{z x}$ of the extracted integral feature for internal features of size and/or the minimum circumscribed size $\mathrm{L}_{\mathrm{ZN}}$ of the extracted integral feature for external features of size. The resulting envelope associated features (lines) have to be parallel (or perpendicular) to the corresponding datum surfaces (or simulated datum features);

e) DGAS is determined as the distance $\mathrm{L}_{z z}$ between two opposing pixels, obtained by the intersection of the two associated mean lines or $\mathrm{L}_{\mathrm{ZX}} / \mathrm{L}_{\mathrm{ZN}}$ for circumscribed associated straight lines and a straight line that is perpendicular to the datum axis. In order to improve the accuracy of the estimate for $\mathrm{L}_{\mathrm{ZZ}}$, the latter can be calculated as the average of $\mathrm{N} \geq 3$ values LzZi $(i=N)$ of features of size that are equally spaced on the image (Fig. 5);

f) If the dimension is determined graphically, one uses the mean straight line, built based on the mean dimension of the minimal MINIMAX zone of the extracted integral element, or uses one of the straight lines that limit the minimal zone. For external dimensional entities this is the outer straight line, and for internal features dimensional entities this is the inner straight line. The approach can be applied without the need of using specialized photographic equipment or specialized software. 


\subsection{Determining the diameter of spherical parts}

The problems of measuring objects having spherical form are among the most interesting and yet the hardest ones to solve in dimensional metrology. It is known that their geometric accuracy is determined by the deviations of the diameter and/or the form deviation from the spherical surface whose total measurement is practically impossible [7]. Nevertheless, PMM can be used for partial and/or compromise solution to the problem. In this case the method needs to be applied consecutively $\mathrm{N} \geq 3$ times, and the desired dimension will be determined as an indirect global calculated size from a homogeneous set local sizes. For this purpose the measurement is performed in the following sequence:

a) the diameter of the sphere $D_{\text {ZZsphere }}$ is determined as a direct global size from a $2 \mathrm{D}$ photo according to the methodology described in item 3.2. The procedure is repeated $\mathrm{N}$ times, where the value of $\mathrm{N}$ is determined by the necessary accuracy of the estimate of the measured diameter;

b) the obtained $\mathrm{N}$ values of $\mathrm{D}$ represent a homogeneous set of local sizes of $\mathrm{N}$ realizations of a local dimension. Using them one can calculate the final indirect generalized calculated dimension of the diameter of the sphere;

c) the diameter of the sphere is calculated as the average, median or mean of the range of the group or is assumed to be the largest one or the smallest one from the group of local dimensions.

\section{Conclusion}

The dimensions obtained when measuring using the photogrammetric measurement method shall be defined as generalized (integral) dimensions obtained as associated using a direct measurement method. Proposed are algorithms for processing the results obtained by the photogrammetric method for manufacturing parts having a general geometrical form of a cylinder, cuboid or sphere. Proposals are made how to process similar results using a graphical method, without the need to use specialized equipment and specialized software.

\section{References}

[1] BDS EN ISO 14405-1:2016. Geometrical Product Specification (GPS) - Dimensional tolerancing - Part 1: Linear sizes.

[2] Effenberger, G. Geometrical Product Specifications (GPS) - ISO 14405-1, the General GPS Standard for Dimensional Tolerancing of Linear Sizes. Q-DAS Web Portal. May 2013.

[3] ISO 286-1:2010. Geometrical Product Specification (GPS) - ISO code system for tolerances on linear sizes - Part 1: Basis of tolerances of deviations and fits.

[4] Metrology and measurement equipment. A reference book in three volumes. (2010). Vol. 2. H. Radev (Ed.), Published by Softrade, pp. 987, ISBN 978-954-334-093-4, Sofia, Bulgaria. (Метрология и измервателна техника. Книга справочник в три тома. Том 2, под общата редакция на проф. Христо Радев - София: Софтрейд, 2010, 987 стр.).

[5] Sakakushev, B.; Kokalarov, M.; Parvanov S. (2018). Perspectives for the application of the photogrammetric method for large-scale details and structures. 28th International scientific symposium "Metrology and metrology assurance 2018”, No. 28, pp. 160 - 162, ISSN 1313-9126.

[6] Sakakushev, B.; Parvanov, S.; Gueorguiev, Tz. (2018) The Real Capabilities of the Photogrammetric and the Stereophotogrammetric Methods for Measurement in Mechanical and Manufacturing Engineering. Proceedings of SOCIOINT 2018, 5th International Conference on Education, Social Sciences and Humanities, Dubai, U.A.E. ISBN: 978-605-82433-3-0.

[7] Sotirov B.; Tonev D. (2020). Measurement of geometric features of spherical objects. International Conference Knowledge-based organization - Sibiu, Romania, Land Forces Academy "Nicolae Balcescu", Conference proceedings Vol. XXVI, No. 3, 2020, pp. 167-172, ISSN: 1843-682X.

[8] Kokalarov, M. (2019). Predetermination of the Uncertainty Budget and Error. Analysis of the Photogrammetric Method in Heavy Machinery. Proceedings of the 30th DAAAM International Symposium. Published by DAAAM International, 2019, Vienna, Austria. ISBN 978-3-902734.

[9] Georgiev G.; Sakakushev B.; Georgieva K. (2015). Study of the applicability of the photogrammetric mehod for measurement of very small dimensions. Proceedings of the University of Ruse, Vol. 54, Series 2. ISSN 1311-3321, Ruse, Bulgaria. (Георгиев, Г., Сакакушев, Б., Георгиева, К. Изследване на приложимостта на фотограметричния метод за измерване на много малки размери. Научни трудове на Русенския университет -2015 , том 54, серия 2.). 